\title{
How do bisphosphonates prevent fractures?
}

\section{Why do fractures occur in osteoporosis?}

In osteoporosis, changed bone remodelling causes loss of bone tissue and a deterioration in skeletal microstructure, which leads to an increase in skeletal fragility and risk of fracture. ${ }^{1}$ These microstructural changes largely consist of a deterioration in skeletal microarchitecture, which affects both trabecular and cortical bone. ${ }^{23}$ In particular, the high bone turnover frequently seen in osteoporosis can produce the following changes: thinning, perforation, and complete removal of trabeculae; expansion of the medullary cavity, thereby reducing cortical thickness; an excess of partially completed cortical bone remodelling units, so increasing the proportion of cortical bone consisting of Haversian canals-that is, cortical porosity.

Recent evidence also suggests that changes in the material composition of bone may also contribute to the increase in skeletal fragility seen in osteoporosis. For example, accumulation of fatigue damage within bone, and a reduction in collagen crosslink concentration, have both been suggested to increase skeletal fragility. ${ }^{45}$ Several other independent determinants of fracture risk have also been identified, such as overall size and shape of the skeleton, ${ }^{67}$ and factors related to the risk of falling such as postural instability. ${ }^{8}$ However, unlike skeletal microstructure, these other factors are not thought to be influenced either by bone remodelling, or by therapeutic agents like bisphosphonates.

\section{Summary of effects of bisphosphonates on bone}

Bisphosphonates have been used to treat patients with osteoporosis since the 1980s. They are similar in structure to inorganic pyroposphate, and bind avidly to bone where they act to inhibit osteoclast activity, ${ }^{9}$ although the precise mechanisms responsible for this action remain to be elucidated. Studies involving biochemical markers of bone turnover in humans have confirmed that bisphosphonates are potent suppressors of bone resorption. ${ }^{10}$ As a consequence of this action, bisphosphonates are effective at preventing further bone loss, and can even lead to substantial increases in bone mass. ${ }^{11}$

These actions of bisphosphonates on bone turnover and bone mass are also associated with a beneficial effect on fracture rate. For example, cyclical administration of etidronate for two to three years has been suggested to reduce the incidence of vertebral fractures in patients with established osteoporosis by about $50 \% .^{12}{ }^{13}$ Two considerably larger studies, which examined the effect of the amino-bisphosphonate alendronate have since confirmed that these agents considerably reduce the incidence of vertebral fractures. ${ }^{115}$ Furthermore, a similar protective effect was observed on the occurrence of fractures at the hip and distal radius. ${ }^{14}$

As bisphosphonates reduce fracture risk, these agents are presumably capable of improving skeletal microstructure. However, while this beneficial effect of bisphosphonates on structure is assumed to be related to their action on bone mass and bone turnover, these latter two parameters only reflect skeletal microstructure indirectly, suggesting the need for a more complete explanation as to how bisphosphonates prevent fractures. In fact, the effects of bisphosphonates and other anti-resorptive treatments on bone mass can be misleading when it comes to predicting their actions on microstructure and fracture risk. For example, after further analysis of the study by Black et $a l,{ }^{14}$ it was suggested that the protective action of alendronate on vertebral fractures far exceeds that predicted by changes in bone mass alone. ${ }^{16}$ This conclusion also applied to the beneficial effect of other classes of anti-resorptive agent on fracture incidence, such as hormone replacement therapy (HRT) and calcitonin. Therefore, to explain how agents like bisphosphonates prevent fractures, it is necessary to study how they influence microstructure using a more direct approach.

\section{Possible effects of bisphosphonates on trabecular microarchitecture}

Several investigators have used histomorphometric analysis of iliac crest bone biopsy specimens to study the effects of bisphosphonates on trabecular architecture. ${ }^{11}$ Taken together, these results suggest that bisphosphonates act to suppress the activation of new sites of bone remodelling, and to reduce the depth of osteoclastic resorption cavities. However, while these effects might be expected to limit any subsequent deterioration in skeletal microstructure by reducing the likelihood of further trabecular perforations, ${ }^{17}$ they do not explain how skeletal microstructure could be improved, and the risk of future fractures actually decreased.

As the major changes in trabecular architecture described in osteoporosis are thought to be largely irreversible, such as loss of trabeculae, ${ }^{2}$ it may be that bisphosphonates exert their beneficial influence on skeletal microstructure through other means. However, even if anti-resorptive agents cannot restore a weakened trabecular architecture, they may be able to significantly reduce skeletal fragility by strengthening those trabecular elements that remain. Consistent with this possibility, a recent histomorphometry study suggests that alendronate is able to 'un-couple' bone formation from bone resorption, because bone resorption after treatment with alendronate for two years was found to be reduced, but mean wall thickness (that is, the amount of bone laid down by individual teams of osteoblasts) to be increased. ${ }^{18}$ However, while this action might be expected to lead to trabecular thickening, whether this accounts for the decrease in skeletal fragility that follows is unclear.

In fact, although previous research has focused on changes in trabecular architecture that occur in patients with osteoporosis, it is not certain whether these are actually responsible for the increased skeletal fragility seen in this condition. For example, in another study, the degree of deterioration in trabecular architecture, as assessed on iliac crest bone biopsy specimens, was not found to predict the occurrence of fragility fractures in patients with osteoporosis. ${ }^{19}$ While there is some in vitro evidence to suggest that trabecular architecture contributes to compressive strength of vertebrae, the relevance of these findings to clinical fractures is unclear because in many of these studies, the cortical shell was removed before loading. ${ }^{20}$

\section{Possible effects of bisphosphonates on cortical} microarchitecture

Cortical bone is also known to contribute a major part to skeletal strength, even at sites like the spine that are rich in trabecular bone. ${ }^{3}$ Thus, it is possible that structural changes in cortical rather than trabecular bone are the major cause of increased skeletal fragility in osteoporosis, and the principal targets for therapeutic agents like bisphosphonates. That cortical bone architecture is a major determinant of skeletal strength seems intuitively 
correct, in view of the fact that mechanical strains are known to be greatest at the periphery of supporting structures.

There is also evidence from clinical studies that suggests that changes in cortical structure contribute significantly to the increase in skeletal fragility associated with osteoporosis. For example, in a study of patients sustaining hip fractures, femoral neck cortical porosity and thickness were found to be respectively increased and decreased in these patients. ${ }^{21}$ In addition, osteoporosis has recently been reported to be associated with thinning of the vertebral cortical shell, as assessed histologically. ${ }^{22}$ Furthermore, in vitro studies have found that cortical thickness and porosity contribute major parts to vertebral compressive strength and femoral neck torsional strength respectively. ${ }^{2324}$

As any tendency for cortical porosity to be increased in patients with osteoporosis probably reflects the high bone turnover that frequently occurs in this condition, this may be potentially reversible after treatment with antiresorptive agents, in contrast with changes in trabecular microarchitecture. Unfortunately, there have been comparatively few clinical studies of the effects of anti-resorptive agents on cortical, compared with trabecular, architecture. In one recent study, however, treatment with HRT was found to significantly reduce cortical porosity, as assessed by analysis of sequential iliac crest bone biopsy specimens. ${ }^{25}$ Thus, if cortical porosity is a major determinant of skeletal fragility, an improvement in this component might explain how anti-resorptive agents such as bisphosphonates are able to prevent fractures in patients with established osteoporosis.

\section{Possible effects of bisphosphonates on bone composition}

Although etidronate can impair skeletal mineralisation, this effect has not been reported after treatment with other bisphosphonates, or when etidronate is given intermittently. ${ }^{1213}$ However, these agents are likely to cause other changes as a consequence of reduced bone turnover leading to an increase in bone age, although the overall impact of these on bone strength is unclear. On the one hand, the comparativley poor degree of bone mineralisation at sites undergoing rapid turnover might be expected to improve; on the other hand, excessive suppression of bone remodelling could theoretically lead to the accumulation of fatigue damage. It is also possible that bisphosphonates change the skeletal composition of collagen, following a recent report that neridronate changes the ratio of collagen crosslink fractions excreted in urine. ${ }^{26}$ However, recently described effects of bisphosphonates on renal collagen crosslink handling $^{27}$ offer an alternative explanation for these findings.

\section{Conclusion}

It seems clear that resorption inhibitors like bisphosphonates are effective at reducing the risk of further fractures in states of established osteoporosis, suggesting that certain aspects of microstructural deterioration associated with osteoporosis are partially reversible. While it seems probable, however, that these microstructural changes involve improvements in certain aspects of skeletal microarchitecture, as yet, these remain unidentified.
Hence, despite the widespread use of anti-resorptive agents in the treatment of osteoporosis, we are still some way from understanding exactly how these drugs prevent fractures.

Rheumatology Unit, Bristol Royal Infirmary,

JONATHAN H TOBIAS

Bristol BS2 8HW

1 Parfitt AM. Quantum concept of bone remodelling and turnover: implications for the pathogenesis of osteoporosis. Calcif Tiss Int 1979;28:1-5.

2 Parfitt AM. Implications of architecture for the pathogenesis and prevention of vertebral fracture. Bone 1992;13:S41-7.

3 Mazees R. Fracture risk: a role for compact bone. Calcif Tiss Int 1990;47:191-3.

4 Burr DB, Forwood MR, Fyhrie DP, Martin RB, Schaffler MB, Turner CH. Bone microdamage and skeletal fragility in osteoporotic and stress fractures. J Bone Min Res 1997;12:6-15.

5 Oxlund H, Mosekilde L, Ortoft G. Reduced concentration of collagen reducible cross-links in human trabecular bone with respect to age and osteoporosis. Bone 1996;19:479-84.

6 Faulkner KG, Cummings SR, Black D, Palermo L, Gluer CC, Genant HK. Simple measurement of femoral geometry predicts hip fractures - the study of osteoporotic fractures. J Bone Min Res 1993;8:1211-7.

7 Gilsanz V, Shultz EE, Loro L, Roe TF, Sayre J, Goodman WG. Smaller cross-sectional area of intact vertebral bodies in women with vertebral cross-sectional area of intact vertebral bodies in
fractures. J Bone Min Res 1993;8 (suppl 1):S326.

8 Nguyen T, Sambrook P, Kelly P, Jones G, Lord S, Freund J, et al. Prediction of osteoporotic fractures by postural instability and bone density. BMJ 1993;307:1111-5

9 Fleisch H. Bisphosphonates: mechanisms of action and clinical applications. In: Peck WA, ed. Bone and mineral research annual I. Vol 1. Amsterdam: Excerpta Medica, 1982: 319-57.

10 Harris ST, Gertz BJ, Genant HK, Eyre DR, Survill TT, Ventura JN, et al. The effect of short term treatment with Alendronate on vertebral density and biochemical markers of bone remodeling in early postmenopausal women. J Clin Endocrinol Metabol 1993;76:1399-406.

11 Francis R. Oral bisphosphonates in the treatment of osteoporosis: a review. Curr Ther Res 1995;56:831-51.

12 Storm T, Thamsborg G, Steiniche T, Genant H, Sorensen O. Effect of intermittent cyclical etidronate therapy on bone mass and fracture rate in women with postmenopausal osteoporosis. N Engl J Med 1990;322:126571 .

13 Watts N, Harris S, Genant H, Wasnich RD, Miller PD, Jackson RD, et al. Intermittent cyclical etidronate treatment of postmenopausal osteoporosis. N Engl J Med 1990;323:73-9.

14 Black DM, Cummings SR, Karpf DB, Cauley J A, Thompson DE, Nevitt $\mathrm{MC}$, et al. Randomised trial of effect of alendronate on risk of fracture in women with existing vertebral fractures. Lancet 1997;348:1535-41.

15 Liberman UA, Weiss SR, Broll J, Minne HW, Quan H, Bell NH, et al. Effect of oral alendronate on bone mineral density and the incidence of fractures in postmenopausal osteoporosis. N Engl J Med 1995; 333:1437-43.

16 Cummings S, Black D, Vogt T. Changes in BMD substantially underestimate the anti-fracture effects of alendronate and other antiresorptive drugs. J Bone Min Res 1996;11 ( suppl 1):S102.

17 Storm T, Steiniche T, Thamsborg G, Melsen F. Changes in bone histomorphometry after long-term treatment with intermittent, cyclic etidronate for postmenopausal osteoporosis. J Bone Min Res 1993;8:199-208.

18 Chavassieux P, Arlot ME, Yates AJ, Roux JP, Meunier PJ. Effects at the basic structure unit of longterm alendronate treatment in postmenopausal ostestructure unit of longterm alendronate treatment
oporosis. Osteoporos Int 1996;6 (suppl 1):251.

19 Flautre B, Hardouin P. Microradiographic aspect on iliac bone tissue in postmenopausal women with and without verebral fractures. Bone 1994;15:477-81.

20 Mosekilde L. Normal vertebral body size and compressive strength: Relations to age and to vertebral and iliac trabecular bone compressive strength. Bone 1986;7:207-12

21 Nishihara A. Femoral neck and iliac bone histomorphometry in femoral neck fracture. Journal of the Japanese Orthopedic Association 1995; 69:156-67.

22 Ritzel H, Amling M, Posl M, Hahn M, Delling G. The thickness of human vertebral cortical bone and its changes in aging and osteoporosis: a histomorphometric analysis of the complete spinal column from thirty-seven autopsy specimens. J Bone Min Res 1997;12:89-95.

23 McCalden RW, McGeough JA, Barker MB, Court-Brown CM. Age-related changes in the tensile properties of cortical bone. J Bone J Surg 1993;75A: 1193-205.

24 Vesterby A, Mosekilde L, Gundersen H, Melson F Mosekilde L, Holme K, et al. Biologically meaningful determinants of the in vitro strength of lumet al. Biologically meaningful determinan
bar vertebrae. Bone 1991;12:219-24.

25 Brockstedt H, Kassem M, Eriksen E. Estrogen prevents cortical bone loss in early postmenopausal women: a histomorphometric study. J Bone Min Res 1996;11 (suppl 1):S448

26 Tobias JH, Laversuch CV, Wilson N, Robins SP. Neridronate preferentially suppresses the urinary excretion of peptide-bound deoxypyridinoline in postmenopausal women. Calcif Tiss Int 1997;59:407-9.

27 Colwell A, Eastell R. The renal clearance of free and conjugated pyridinium cross-links of collagen. J Bone Min Res 1996;12:1976-80. 